\title{
Determinantes del subempleo y la informalidad para Honduras 2012
}

\author{
Sergio Francisco Zepeda*, Ela Koritza Díaz *, Zairy Yajaira Rivera *, \\ Héctor Alvarado **
}

\section{RESUMEN}

Los mercados laborales de Honduras muestran una marcada heterogeneidad, característica presente en todas las regiones latinoamericanas; resultando que los empleados de los distintos sectores no gocen de todos los beneficios no salariales que brinda el Estado y convivan con grupos que no los posean. De aquí procede en la actualidad el estudio de las condiciones laborales y la calidad del empleo, por su relevancia en la elaboración de políticas públicas y privadas, con el fin de reducir las diferencias marcadas entre empleados de un sector y otro.

Por esta razón, la informalidad y el subempleo cobran importancia al ser medidas de la baja calidad del empleo; una desde la óptica de la oferta laboral y la otra desde la demanda laboral. Así como conocer las inclinaciones de los individuos a optar por estas propuestas que no maximizan los beneficios esperados de sus empleos.

Palabras Clave: informalidad, subempleo, modelo biprobit, ephpm, calidad del empleo, determinantes.

\section{ABSTRACT}

In Honduras, labor markets show a strong heterogeneity feature, which is present in all Latin American Regions, thus proving that all employees from different sectors do not enjoy all nonwage profits provided by the State and therefore does not allow them to share with the less fortunate groups. From here on, the quality of employment and the working conditions are studied due to their relevance in the making of public and private political policies, in order to reduce gaps among employees from different sectors.

\footnotetext{
*Universidad Nacional Autónoma de Honduras. Facultad de Ciencias Económicas. Escuela de Economía. "Universidad Nacional Autónoma de Honduras. Facultad de Ciencias Económicas. Escuela de Economía Asesor.hector_25@yahoo.com
} 
For this reason, informality and sub employment become an important measure of low quality employment, seen from the standpoint of labor supply and demand. Therefore, it seeks to find out why the individuals prefer to choose labor options that will not maximize the expected benefits of the employees.

Key Words: informality, underemployment, probit, PMHS, job quality, determinants. 


\section{INTRODUCCIÓN}

Las Naciones Unidas (2010) afirman que durante el período 2003-2008 en el que la economía hondureña experimentó el crecimiento más alto en toda su historia, 5.8\% en promedio, no se generaron los suficientes empleos dignos y productivos para reducir de manera significativa el subempleo invisible. Según el Banco Mundial (2012), la crisis económica de 2008-2009 deterioró la calidad de los empleos que se ofrecen en Honduras, porque se destruyó gran cantidad de empleos formales en las áreas urbanas y rurales, trayendo consigo un aumento en la desigualdad del ingreso, quedando evidenciada la alta vulnerabilidad del mercado laboral en Honduras, traducido en un incremento de la informalidad y la subocupación.

A pesar de lo anterior, no se ha visto el interés de las autoridades por hacer las reformas convenientes en el mercado laboral y en las leyes laborales vigentes; es por ello que, las condiciones actuales del mercado laboral hondureño no están fundamentadas en base a estudios económicos, lo que provoca que su niveles se mantengan altos; en específico lo referente al subempleo e informalidad.

El Banco Mundial (2012) estimó que el 80\% de la población hondureña se encuentra en la informalidad y que uno de cada tres ocupados es subempleado, es decir los empleos en Honduras no responden a las necesidades de la población ocupada y de los jóvenes que se incorporan a ella cada año, como consecuencia, el detrimento de la calidad de los empleos y la falta de oportunidades ha provocado una disminución de las condiciones de vida de la población más vulnerable y esto explica porque los niveles de pobreza de la población no se han reducido a pesar de tener estrategias orientadas a minimizarla.

En la Constitución de la República que entró en vigencia el 20 de enero de 1982, en su artículo número 127 señala que "Toda persona tiene derecho al trabajo, a escoger libremente su ocupación y a renunciar a ella, a condiciones equitativas y satisfactorias de trabajo y a la protección contra el desempleo". Si bien es cierto que el Congreso de la República tuviera la intención de cumplir todas las garantías que se ofrecen en este artículo; el Estado en sí mismo no podría lograrlo; lo cierto es que es necesario que se equilibre la oferta y demanda de trabajo, de lo contrario, siempre habrán personas que estarán fuera del mercado laboral a pesar que deseen ser parte de él.

Es necesario resaltar que existen desequilibrios en el mercado de trabajo hondureño, porque la demanda de trabajo es menor que la oferta de trabajo lo que se traduce en desempleo. Estas irregularidades propician la existencia de empleos 
de baja calidad, porque el mercado no puede absorber satisfactoriamente toda la fuerza de trabajo disponible. Según Uribe, Ortiz, y García (2008), los indicadores más eficientes para la medición de la calidad de los empleos son la informalidad y el subempleo, porque son generales y comparables, pero son variables que tradicionalmente se han analizado por separado. En Honduras, los instrumentos de medición de estadísticas del mercado laboral utilizados por el gobierno se enfocan en su mayor parte a captar el subempleo y no la informalidad. Ambas variables representan valores diferentes, pero comparten determinantes en común, lo que incide en que los individuos decidan aceptar un empleo formal o informal en el mercado. Esto implica que su remuneración puede no ser la que él esperaba o que no cuente con protección social, a pesar que la Constitución de la República afirme que los individuos tienen derecho a escoger libremente su empleo, ellos siempre se enfrentan a restricciones que están influenciadas por la estructura en que están conformados los mercados de trabajo.

Para el desarrollo de esta investigación, se plantea como objetivo general identificar los determinantes del subempleo y la informalidad en Honduras; y como objetivos específicos: determinar la relación entre el subempleo y la informalidad, e identificar los determinantes que explican la diferencia entre el subempleo y la informalidad. Este estudio es un acercamiento a lo que puede ser una nueva metodología para la medición de la calidad de los empleos en Honduras, con el fin de proporcionar una herramienta para futuros investigadores y que contribuya al desarrollo de políticas que tengan como base las estimaciones necesarias para la toma de decisiones. El análisis propone una política de generación de nuevos empleos de buena calidad que debe acompañarse con el desarrollo de capital humano apto para desempeñarse en esos puestos de trabajo, de lo contrario, difícilmente se verán mejoras o resultados de las políticas traducidos en aumentos para el bienestar de losindividuos.

Se considera que el subempleo es un problema de la oferta de trabajo, porque "caracteriza la calidad del empleo desde la perspectiva subjetiva de la oferta laboral y se refiere a una subutilización de las capacidades productivas de los trabajadores o una remuneración inadecuada" (Uribe et al, 2008, p. 2). Los mismos autores, afirman que la informalidad es un problema de la demanda de trabajo porque refleja las características objetivas de los empleos, relacionadas a la capacidad del individuo para generar ingresos, lo que quiere decir, que la informalidad por definición, se refiere a empleos que baja productividad y por lo tanto de bajos ingresos.

El análisis en conjunto del subempleo y la informalidad, al ser el primero un problema de la oferta laboral y el segundo de la demanda laboral, permite conocer 
los elementos que pueden estar influyendo en que los valores de estas dos variables no coincidan. Entonces, la importancia de realizar este estudio es que se pretende hacer una identificación de las condiciones de empleo en Honduras, traducida en los indicadores de subempleo y de informalidad con el fin de ser una propuesta con una perspectiva diferente para lograr una caracterización más asertiva de los determinantes que pueden estar influyendo en la decisión de un individuo por subemplearse o sumarse a las filas de la población informal.

\section{METODOLOGÍA}

Se ha recurrido al enfoque cuantitativo analítico para el desarrollo de este tema de investigación, el cual se basa en el uso de técnicas de análisis estadístico y econométrico, apoyados en los datos del Instituto Nacional de Estadística (INE) de la Encuesta Permanente de Hogares de Propósitos Múltiples (EPHPM) de mayo de 2011, para inferir estadísticamente de las variables que se analizará en este prospecto. La bibliografía revisada para este estudio sirvió de guía para definir operativamente las variables -subempleo e informalidad-, las cuales se midieron para el caso de Honduras y así lograr establecer la relación entre los determinantes de estas variables, para tener como fin que sirva de punto de partida en las futuras investigaciones acerca de estos problemas laborales.

Este estudio se abordó desde dos perspectivas: 1.-Se expone al subempleo y la informalidad como indicadores independientes el uno del otro, para lograr con ello la cuantificación de su impacto en los mercados laborales y así poder proponer los determinantes que más inciden en esta relación, medida a través del número de subempleados (tanto visibles como invisibles) y el número de informales; 2.- Se expone el análisis ligado a ambas variables para cuantificar el grado de correlación en los determinantes que comparten ambos problemas, y con ello especificar un modelo que se adapte al conjunto de determinantes que pueden incidir.

Es importante resaltar que el uso de la EPHPM que realiza el INE sirvió como fuente de información secundaria, ya que este tipo de encuesta proporciona los datos necesarios acerca de las variables de estudio; pero se debe considerar que la encuesta permanente de hogares no recoge la información directa sobre informalidad o formalidad de los individuos en el trabajo por lo que se debe hacer una aproximación con base en las recomendaciones de la Organización Internacional del Trabajo (OIT) el cual puede definirse en términos generales como el conjunto de unidades dedicadas a la producción de bienes o a la prestación de servicios con la finalidad primordial de crear empleos y generar ingresos para las personas que participan en esa actividad. Estas relaciones de empleo, se basan en 
el empleo ocasional, el parentesco o las relaciones personales y sociales y no en acuerdos contractuales que supongan garantías formales. (OIT, 2003).

El modelo econométrico más conveniente en la realización de este trabajo es el que involucra el uso de un modelo Probit bivariado, "este modelo de extensión natural consiste en considerar más de una ecuación y permitir que los errores del modelo estén correlacionados" (Greene, 1999). La ventaja que presenta este modelo es que se elimina los errores auto correlacionales seriales, permitiendo que las perturbaciones aleatorias de los errores de este sistema de ecuaciones tengan una covarianza distinta a cero, permitiendo analizar el subempleo y la informalidad como una elección independiente a las condiciones del mercado laborales existentes.

Este modelo econométrico permite que la informalidad y el subempleo conformen la variable dependiente, cada una de ellas tomando valores de uno o cero para mostrar su condición de informalidad o subempleo al momento de simular el modelo. Entre los determinantes que se usarán en esta investigación se puede mencionar: nivel educativo más alto que alcanzó, jefe de hogar, casado, género femenino, posición ocupacional en el empleo actual, tamaño de la empresa del empleo actual, rama de actividad del empleo actual. Otro aporte que brinda el uso del modelo biprobit en esta investigación, radica en que permite estimar el impacto conjunto de los determinantes de la informalidad y el subempleo, ante la posibilidad de elección de un empleo de baja calidad en Honduras.

\section{RESULTADOS}

Los resultados de las estimaciones del modelo de probabilidad bivariada arrojan que las características socioeconómicas del individuo tomadas en cuenta como el nivel educacional, el estado civil, su posición como jefe de hogar y el género, son altamente significativas en cuanto a la probabilidad conjunta de ser informal y subempleado. Para tener una visión más clara de estos determinantes, se estimó tres modelos diferentes, uno que midió la probabilidad conjunta de ser subempleado e informal, otro en donde se puede observar la probabilidad de ser formal y subempleado y el último donde se analiza la probabilidad de ser no subempleado de informal.

\section{CONCLUSIONES}

- En los cálculos realizados se demostró que los determinantes socioeconómicos como el nivel educativo, el estado civil, el género, el ser jefe de hogar; influyen ineludiblemente en la decisión del individuo al momento de elegir el empleo. 
- Las personas que tienen un mayor nivel de educación son menos propensas a emplearse en un trabajo informal o subemplearse, y ocurre lo contrario con los individuos que no alcanzan un nivel educativo alto. Por esta razón, ellos tienden a optar por empleos de baja calidad.

- En cuanto al género, el estudio arrojó que en Honduras siguen existiendo desigualdades en cuanto a hombres y mujeres, ya que ellas por lo general son excluidas del mercado laboral por la falta de oportunidades que provocan que muchas veces se dediquen a actividades domésticas, en lugar de estudiar una carrera universitaria o emplearse en un trabajo formal.

- En cuanto a la rama de actividad, los individuos del sector construcción suelen ser informales y no subempleados, contrario al sector manufacturero donde son en su mayoría formales y subempleados. Para los trabajadores domésticos, cuenta propia que no contratan mano de obra temporal y trabajadores familiares no remunerados, las probabilidades de ser subempleados e informales son muy altas, reflejada en sus efectos marginales mostrados en los cuadros.

- En los sectores que se disminuye la probabilidad de ser informal y subempleado son el comercio, transportes, servicios financieros, comunales y comunicaciones, porque se observa que son rubros en los que las empresas son en su mayoría formales.

- No se pudo analizar el sector agrícola en el modelo planteado por su estrecha relación con las ramas de actividad anteriormente descritas, ya que generaba problemas de multicolinealidad.

- En cuanto a la posición ocupacional en el empleo actual, como era de esperarse las personas que trabajan en el gobierno su probabilidad de ser informal 0 subempleado es nula, pero en cambio en la empresa privada la alta heterogeneidad de los puestos de trabajo provoca que las personas se subempleen o decidan ser informales. De igual forma, los trabajadores domésticos y los cuentapropistas que contratan mano de obra temporal, son muy propensos a ser informales o subempleados.

- A pesar de que los organismos internacionales definen como informales a las empresas que tienen cinco o menos trabajadores, se encontró en el estudio que esto no es del todo cierto. La definición falla porque hay trabajadores por cuenta propia que son profesionales o técnicos, y empresas que tienen más de seis trabajadores que no les proveen un seguro social o que no le pagan el salario mínimo correspondiente a sus trabajadores. Lo que quiere decir que el tamaño de la empresa como variable proxy de la informalidad, no es la más adecuada.

\section{BIBLIOGRAFÍA}

- Banco Mundial. (2012). Mejores Empleos en Honduras, El rol del capital 
Humano. Departamento Humano Región de América Latina y el Caribe.Recuperado el 29 de septiembre de 2012, de http://wwwwds.worldbank.org/external/default/WDSContentServer/WDSP/IB/2012/10 /15/000425962_20121015151919/Rendered/PDF/729210ESWOWhit00emple os0en0Honduras.pdf

- Bardales, J. (2011). Informalidad y subempleo: evidencia microeconómica para el paso peruano. Horizonte económico, 1(2), (6-17). Recuperado el 29 de septiembre de 2012, de http://fce.unac.edu.pe/Files/a1.pdf

- Castellar, C. E., \& Uribe, J. I. (2001). Determinantes de la participación en el mercado de trabajo en el área metropolitana de Cali en diciembre de 1998. Recuperado el 2 de 10 de 2012, de http://www.researchgate.net/publication/ 5006987_Determinantes_de_la_Participacin_en_el_Mercado_de_Trabajo_ del_rea_Metropolitana_de_Cali_en_Diciembre_de_1998

- Castellar, C., \& Uribe, J. I. (2002). La Participación en el mercado de trabajo, componentes micro y macroeconómico. Recuperado el 29 de septiembre de 2012, de http://economialaboral.univalle.edu.co/Ponenciapartifinal.pdf

- Figueroa, C. (2010). Determinantes de la informalidad laboral y el subempleo en las áreas metropolitanas de Barranquilla, Cartagena y Montería. Serie de documentos del instituto de estudios económicos del Caribe, 32. Recuperado el 27 de septiembre de 2012, de http://odesdo.org/servlet//imagess/0/ adjuntos/c112715cc5f426775b08a1b5ac050b11.pdf

- García-Ubaque, J., Riaño-Casallas, M., \& Benavides-Piracón, J. (2012). Informalidad, desempleo y subempleo: un problema de salud pública. Revista de salud pública, 14 (1), (138-150). Recuperado el 30 de septiembre de 2012, de http://www.scielo.org.co/pdf/rsap/v14s1/v14s1a12.pdf

- Gomez, L. G. (2007). La Informalidad en la economía, algo indiscutible. Semestre económico, 19 vol.10, (47-67). Recuperado el 30 de septiembre de 2012, de http://redalyc.uaemex.mx/redalyc/pdf/1650/165013672003.pdf

- Greene, W. H. (1999). Analisis Econometrico (Tercera ed.). Madrid: Pearson EDUCATION.

- Martinez Coll, J. C. (2007). El modelo neoclásico del mercado de trabajo y el desempleo. Recuperado el 29 de septiembre de 2012, de http://www.eumed.

net/cursecon/14/modelo_neoclasico_del_desempleo.htm

- Naciones Unidas. (2010). Objetivos del Milenio Honduras 2010. San José, Costa Rica.

- Naciones Unidas. (2010). Objetivos de Desarrollo del Milenio. El Progreso en América Latina y el Caribe Hacia los Objetivos del Milenio. Desafíos para lograr la igualdad.

- Organizacion Internacional del Trabajo [OIT]. (2003). Resolución sobre las 
estadísticas del empleo en el sector informal, adoptada por la decimoquinta Conferencia Internacional de Estadísticos del Trabajo. Recuperado el 29 de septiembre de 2012, de Organizacion Internacional del Trabajo: http://www.ilo.org/public/spanish/bureau/stat/download/res/infsec.pdf

- Perry, G., Maloney, W., Arias, O., Fajnzylber, P., \& Mason, A. (2007). Informalidad: escape y exclusión. Estudios del Banco Mundial sobre América Latina y el Caribe. Recuperado el 29 de septiembre de 2012, de http://siteresources.worldbank.org/INTLACINSPANISH/Resources/SP_la cf_Overview.pdf

- PNUD. (2009). Informe sobre desarrollo humano Honduras 2008-2009. San José, Costa Rica: Imprenta Lil, S.A.

- PNUD. (2012). Informe sobre desarrollo humano Honduras 2011. San José, Costa Rica.Imprenta Lil, S.A.

- Programa de las Naciones Unidas para el Desarrollo (PNUD). (marzo de 2012). Informe sobre Desarrollo Humano Honduras 2011. San José, Costa Rica: Imprenta Lil, S.A.

- Saravia, F. F. (2011). El bono demográfico en riesgo: una mirada desde las tendencias de la pobreza, la desigualdad y la informalidad laboral. Revista población y desarrollo: argonautas y caminantes, 7, vol.7, (9-15). Recuperado el 30 de septiembre de 2012, de http://www.lamjol.info/index.php/PDAC/article /view/687

- Uribe Garcia, J. I., Ortiz Quevedo, C. H., \& Garcia Cruz, G. A. (2008). Informalidad y subempleo en Colombia: Dos caras de la misma moneda. Bogota. Recuperado el 10 de septiembre de 2012 de http://www.docstoc.com/docs/3253709/INFORMALIDAD-Y-SUBEMPLEOEN-COLOMBIA-DOS-CARAS-DE-LA-MISMA

- Uribe, J. I., \& Ortiz, C. H. (2006). Informalidad laboral en Colombia 1988-2000. Evolución Teorías y Modelos. Cali, Colombia: Universidad del Valle.

- Uribe, J. I., Ortiz, C. H., \& Correa, J. B. (2004 ). Determinantes de las decisiones de mercado laboral: la decisión de ser informal en Colombia 1988-2000. Centro de Investigaciones de la Universidad del Valle, Documentos de Trabajo, N79.

- Uribe, J., Ortiz, C., \& y García, G. (2008). Informalidad y subempleo en Colombia: dos caras de la misma moneda. Cuadernos de administración, 37, vol.21, (211241). Recuperado el 20 de septiembre de 2012, de http://www.scielo.org.co /pdf/cadm/v21n37/v21n37a10.pdf

- Zambrano, A. (2010). Análisis de la informalidad y el subempleo en Ibagué 2008. Recuperado el 23 de septiembre de 2012, de https://docs.google.com 